\title{
Development of image based modeling for determination of oil content and days estimation for harvesting of fresh fruit bunches
}

\begin{abstract}
In this study, the relationship of oil extraction rate (OER) and fruit ripeness will be determined. The sample of oil palm fruits was collected from the unripe until the overripe stage and the oil content of the mesocarp for fresh fruit bunches (FFB) was extracted by using bunch analysis procedure to get the oil extraction rate. Using the same samples of FFB, the pixel value of images which measure in hue, was determined by developed image analysis software. The images were captured under an outdoor environment in an oil palm plantation. The sunlight intensity of environment was recorded using Extech light meter at various times of the day from morning to afternoon in the oil palm plantation. The result of the experiment that showed a good relationship was found between the oil content of FFB with its image pixel values. The mathematical model was developed in determining the optimum days for FFB harvesting.
\end{abstract}

Keyword: Maturity prediction; Outdoors vision system; Hue pixel values; Oil palm FFB 